\title{
Museología Experimental. Hacia un método práctico
}

Experimental museology. Towards a practical methodology

\section{Melissa Aguilar Rojas}

\section{(2) OpenEdition}

1 Journals

Edición electrónica

URL: http://journals.openedition.org/iss/1980

DOI: $10.4000 /$ iss. 1980

ISSN: 2306-4161

Editor

ICOM - International Council of Museums

\section{Edición impresa}

Fecha de publicación: 1 agosto 2020

Paginación: 79-93

ISBN: 978-2-491997-11-3

ISSN: 2309-1290

Referencia electrónica

Melissa Aguilar Rojas, « Museología Experimental. Hacia un método práctico », ICOFOM Study Series

[En línea], 48-1 | 2020, Publicado el 01 agosto 2020, consultado el 08 agosto 2020. URL : http://

journals.openedition.org/iss/1980; DOI : https://doi.org/10.4000/iss.1980 


\title{
Museología Experimental. Hacia un método práctico
}

\section{Melissa Aguilar Rojas UNAM, Ciudad de México, México}

\author{
RESUMEN
}

El texto incentiva la investigación e implementación de prácticas museológicas experimentales con un enfoque enactivo, con el objetivo de facilitar experiencias significativas en los visitantes del museo. Se analizan aspectos que justifican la necesidad de despertar agencia en la persona visitante desde la apropiación tecnológica y el pensamiento crítico, especialmente en el contexto latinoamericano.

Palabras clave: museología experimental, tecnología, agencia, asimilación, visitantes.

\section{ABSTRACT}

\section{Experimental museology. Towards a practical methodology.}

The text promotes the research and implementation of experimental and practical museological practices with an enactive approach aiming to foster significant experiences in the museum visitors. An analysis of key aspects to justify the need to awake agency in the visitor is presented using technological appropriation and critical thinking focused

in the Latinamerican context.

Keywords: experimental museology, technology, agency, assimilation, visitors. 
En esta nueva década, la especie humana se encuentra en un momento drástico de reconfiguración para garantizar la calidad de vida ante las crisis políticas, ambientales y culturales que nos retan. El museo, como elemento clave de la sociedad, no se escapa de la adaptación para su supervivencia. ¿Cómo sobrevive el museo en su carácter de institución actualmente? (Aguilar, 2019, p.I6) Dentro de las múltiples respuestas a este cuestionamiento, resaltaremos a lo largo del texto la necesidad por retomar una museología experimental, a sabiendas de que han habido multiples propuestas de experimentación de diversas naturalezas hace bastante tiempo.

Quizás uno de los ejemplos más dinámicos para la memoria de la museología es la creación de la documenta. Esta bienal, surgió como iniciativa de Arnold Bode para mostrar el arte considerado como degenerado y por tanto ilegal por el régimen nazi durante la segunda guerra mundial. Las obras confiscadas y posteriormente liberadas, "obras renegadas, fugitivas", formaron parte de la primera documenta en el año 1955 en la ciudad de Kassel, Alemania, donde se continua celebrando hoy día.

La variedad de piezas, la aparente discrepancia temática, temporal, justificada como un acto político desde el arte, se traduce en una experimentación activa en el espacio museográfico. Con cada edición, documenta trae nuevas propuestas artísticas relevantes no sólo por las obras en sí mismas, sino por sus formatos de montaje y puntos de vista curatoriales.

Cada documenta adquiere su carácter de las ideas y conceptos de su director artístico, y por ello es no solamente un forum para las tendencias actuales en el arte contemporáneo, pero también un lugar donde se prueban conceptos innovadores y marcan estándares expositivos. (documenta gGmbH, 2020)

En el caso de Latinoamérica, ¿cómo son estas prácticas museológicas experimentales? ¿Qué otras estrategias pueden funcionar y bajo cuales enfoques que lleven a la innovación?

Los siguientes párrafos tienen como objetivo describir un enfoque experimental, proponer actividades para probar dicho enfoque y presentar ejemplos que se 
acercan al modelo que se intenta desarrollar, como parte de la investigación de la autora sobre inmersividad ${ }^{\mathrm{T}}$, arte y tecnología.

Para promover un enfoque enactivo a la exposición y hacia la asimilación no convencional - es decir, una asimilación que se desliga del modelo mental usual (Piaget, 1952) - como una técnica de museología experimental, es necesario interferir y hackear el circuito regular de información. Añadir cambio y variaciones al sendero cognitivo de los visitantes a través de diferentes técnicas aplicadas en el espacio expositivo. Pero antes de entrar en el desarrollo de esta idea es primordial conocer de dónde proviene este enfoque enactivo, cuáles de sus elementos se consideran aplicables a esta propuesta y por qué puede ser la enacción una estrategia provechosa para la experiencia de quien visita el museo.

Enacción significa hacer emerger, hacer existir, "situar la cognición como acción corporizada" (Varela, Thompson \& Rosch, 1992) dependiendo del individuo en un entorno determinado. Desde los años I99o, personas de ciencia se esmeraron por desafiar las teorías cognitivas que estipulaban, a grandes rasgos, que el conocimiento o la aprehensión se daba únicamente en el cerebro. Al contrario, la enacción propone una manera alternativa de conocer, donde los comportamientos son antecedidos y condicionados por el entorno.

El uso del concepto y la palabra enactivo o enactiva en esta propuesta se nutre desde tres análisis de distintos autores y disciplinas. Primero, desde la investigación sobre Museología Enactiva de Daniel Schmitt y Olivier Aubert, en los textos "Por una aproximación enactiva en la museología (2015)" una metodología para comprender la micro-dinámica de la experiencia de los visitantes de los museos"3 (Schmitt \& Aubert. 2017).

La investigación de Schmitt y Aubert, presenta una de las primeras aplicaciones del acercamiento enactivo en el campo de la museología. Esta referencia conduce a la raíz teórica de la enacción en las ideas propuestas por Varela, Thompson y Rosch (1992), pertenecientes al campo de las ciencias cognitivas y a los sistemas robóticos. El tercer análisis es desde el trabajo de Ezequiel A. Di Paolo en psicología, titulado "El enfoque enactivo: bocetos teóricos de la célula a la sociedad" (2013). El autor coincide con Schmitt y Aubert en el planteamiento que califica todo comportamiento como un fenómeno relacional que antecede a cualquier acción o movimiento del cuerpo. "No se piensa al mundo como condición previa para una acción efectiva" (Di Paolo, 2013). La cognición no sucede solamente en el cerebro, sino que se da en todo el cuerpo y se condiciona por el entorno.

\footnotetext{
I. Lo inmersivo se refiere a estar dentro de algo, sumergirse, rodearse. Es por ello que se argumenta que la realidad física es de por sí inmersiva: nos rodea, nos contiene, estamos en ella aún cuando nuestros pensamientos estén en otras realidades (digitales, mentales, etc.) y es a través de la interacción que nos conectamos a ella. (Aguilar, 2019)

2. Trad. Por M. Aguilar 2020.

3. Trad. Por M. Aguilar 2020.
} 
Tomamos en cuenta aquí una visión en la que el museo se constituye como sistema abierto (Varela \& Maturana, 1994), en el que existen mecanismos internos y externos que le modifican y mantienen. El museo depende de sus visitantes para mantenerse, los cuales pueden considerarse factores externos que permiten el funcionamiento del sistema museal. Es necesario comprender la relación de los visitantes con la versión de realidad que perciben durante la visita.

A continuación, se explica lo que destacamos de las tres investigaciones para ubicar el contexto del enfoque enactivo en esta investigación y en una posible derivación de la Museología Experimental.

\section{Desde las investigaciones de Varela, Thompson y Rosch (1992)}

La cognición, en su sentido mas abarcador, consiste en la enactuación de un mundo - en hacer emerger un mundo- mediante una historia viable de acoplamiento estructural. (1992, p.238)

Cada exposición es la emergencia de un mundo. Este mundo enactuado o creado artificialmente está conformado, a grandes rasgos, por los objetos de arte y la línea narrativa perfilada por la curaduría, que la institución elabora. Una vez la exposición se abre al público, los visitantes también se convierten en actores y actrices que habitan y aportan al mundo mediante sus interacciones en la exposición. Dentro de esta propuesta nos interesan las Historias viables de acoplamiento estructural (H.V.A.E.) (Varela, Thompson, Rosch, 1992) de los visitantes, creando una enactuación de mundo dinámica, cambiante, temporal y por tanto experimental.

Las historias viables de acoplamiento estructural tienen la característica de ser proscriptivas, es decir, toda acción que se realice por el sistema se permite si mantiene la integridad del sistema, su esencia o su linaje.

Tomamos en cuenta que, en la investigación de Varela y compañeros, el concepto de inteligencia se redefine; deja de ser la capacidad para resolver un problema y se transforma en la capacidad de ingresar en un mundo compartido de significación (I992 p.240). En el caso de esta propuesta metodológica, el contexto inicial es la exposición de arte, que se vuelve el mundo compartido (enactuado) de significación mediante la implementación o fabricación de artefactos cognitivos inteligentes (Varela, Thompson, Rosch, 1992) también llamados dispositivos, por parte de los visitantes. La fabricación de artefactos en el contexto de un taller o actividad de sala vendría a ser parte del proceso de enactuación de mundos mediante las H.V.A.C. de cada visitante y la inteligencia residiría en la integración de visiones individuales de los visitantes en un mundo compartido de significación (visión institucional y visión de los visitantes). 
La mediación es clave para mantener la integridad del sistema y garantizar la enacción con actividades proscriptivas. Es por ello que se propone una metodología que la garantice en la segunda parte del texto.

\section{Desde la investigación sobre Museología Enactiva de Daniel Schmitt (2016)}

La museología estudia una relación específica entre el hombre y la realidad, caracterizada como una documentación de lo real para la aprehensión directa desde lo sensible. Esta relación operativa, se apoya sobre fragmentos de la realidad, en cosas (objetos) reales, y en situaciones comunicativas a través de la intención de los visitantes. ${ }^{1}$

(D. Schmitt, 2016, p 107)

99

Aquí vemos como la función de los museos incluye mostrar historias vinculantes con ciertas situaciones sociales de importancia para la colectividad. Al mostrarnos fragmentos de la realidad, contribuyen al despertar de las personas $\mathrm{y}$ a generar conciencia respecto al discurso que se exponga.

La investigación de Schmitt propone una comprensión profunda del comportamiento de los visitantes y anota un problema que pretendemos solventar: muchos han sido los estudios de visitantes realizados desde una observación "formal", normalmente utilizando herramientas de evaluación como cuestionarios antes y después de la visita. Pero esto no es suficiente para definir si la experiencia fue significativa. Una aproximación enactiva, al contrario, se interesa en investigar cómo la dinámica del cuerpo y la cognición del visitante en el espacio expositivo se pueden comprender por medios más dinámicos y precisos. Esto obedece a la premisa destacada por Schmitt de que un comportamiento es anterior a cualquier movimiento relativo del cuerpo en el espacio donde el investigador tiende a hacer una descripción. (Schmitt, 20I6, p. 48) 2 .

\section{Desde "El enfoque enactivo: bocetos teóricos de la célula a la sociedad" por Ezequiel A. Di Paolo}

El autor enumera los conceptos relevantes para esta investigación y que justifican al enactivismo como un enfoque válido para la museología experimental: autonomía (del visitante, en este caso), la búsqueda de sentido (experiencia significativa en el museo), la corporarización (asimilación de información desde el cuerpo), emergencia y experiencia (estos dos últimos aún más enfocados en la participación creativa de los visitantes). (Di Paolo, 2013, p.2).

\footnotetext{
I. Trad. M. Aguilar

2. Trad. Por M. Aguilar 2020
} 
Destaca la participación activa el en el mundo (Di Paolo, 20I3 p.2); la cual en esta propuesta se aplica a las dinámicas colaborativas de creación que se explican más adelante. Así como la búsqueda de sentido definida como la interacción significativa y sujeta a normas entre el agente y su mundo (Di Paolo, 20I3 p.5); equivalente en este trabajo a la incorporación de las versiones individuales de los visitantes a la narrativa principal generada por la institución.

Una vez que hemos situado un poco a los lectores en la necesidad del enfoque enactivo para esta propuesta, continuaremos profundizando en la museología experimental propiamente. Las aportaciones de Bruno B. Soares, son clave para guiar hacia las características propuestas en relación a la enacción y a una experiencia significativa en los visitantes del museo.

\section{Museología Experimental}

La experimentación es la palabra clave para la investigación del museo a partir de la sociabilidad de lo museal o envisionando la agencia de ese dispositivo. (Brulon Soares, 2017.)

La cita anterior contiene dos relaciones importantes para la museología experimental: la parte social y la relación que implica agencia. Ambas relaciones, así como la temporalidad en que se proponen como bases para la experimentación, corresponden con características de prácticas museológicas pertenecientes a la Nueva Museología. Como recuerda Galindo Monteagudo (2019):

(...) no olvidar todo aquello que la Nueva Museología forjó, este interés en el público asistente que desde los años sesenta intenta hacer del museo un lugar para todos y que comenzó con los estudios y la crítica al museo como espacio hegemónico de Bourdieu y Darbel (2003) y Adorno (1967).

Esta sociabilidad de lo museal es la transmisión de conocimientos al visitante sobre el museo, sus mecanismos y objetos. En el enfoque por una museología experimental, esta sociabilidad tiene que ver con las formas alternativas de compartir conocimiento en los museos.

¿Cómo son estas formas o prácticas alternativas para socializar efectivamente lo museal? Como primer supuesto, solo a través de la práctica y la observación en el espacio museográfico podríamos corroborar y definir dichas formas. Pero tomando en cuenta las tendencias sociales contemporáneas de interacciones humanas influenciadas por las crecientes tecnologías de la información, pode- 
mos proponer ciertas características para las prácticas museológicas experimentales, las cuales se detallan a continuación.

\section{Tipo de Evaluación:}

Medidas o evaluadas con métodos cualitativos en los que la visión subjetiva de los visitantes se ponga en alto valor. Tomando en cuenta que la visita al museo será distinta para cada persona según su historia personal, una evaluación que deje bastante espacio para la opinión podría revelar resultados inusuales y provechosos para las nuevas prácticas museológicas. Esta evaluación, destacaría las emociones del visitante con el fin de conocerle más íntimamente y valorar la importancia de la visita al museo en su vida.

\section{Relevancia de los visitantes:}

Los visitantes son imprescindibles porque que nos interesa demostrar que son un elemento esencial para completar el circuito de una exposición. Esta propuesta busca vincular con visitantes de manera activa, prefiriendo un grupo reducido de visitantes con disposición a participar en un taller para lograr una experiencia significativa trascendental. No interesa aumentar la visitación al museo. Se prefiere la calidad ante la cantidad. Si bien esta propuesta es orientada a la experiencia del visitante, los objetos mostrados en el museo son, en segundo orden, fundamentales para el desarrollo de la propuesta práctica que se detallara más adelante.

\section{Documentación-Registro:}

Es fundamental generar un registro multimedia de la experiencia en todas las etapas del proceso. Esta propuesta es una investigación en las artes, basada en la definición de Borgdorff (2010):

Se refiere a la investigación que no asume la separación de sujeto $y$ objeto, $y$ no contempla ninguna distancia entre el investigador y la práctica artística, ya que ésta es, en sí, un componente esencial tanto del proceso de investigación como de los resultados de la investigación. Este acercamiento está basado en la idea de que no existe ninguna separación fundamental entre teoría y práctica en las artes. Después de todo, no hay prácticas artísticas que no estén saturadas de experiencias, historias y creencias; $y$ a la inversa, no hay un acceso teórico o interpretación de, la práctica artística que no determine parcialmente esa práctica, tanto en su proceso como en su resultado final. Conceptos y teorías, experiencias y convicciones están entrelazados con las prácticas artísticas y, en parte por esta razón, el arte es siempre reflexivo. De ahí que la investigación en las 
artes trate de articular parte de este conocimiento expresado a través del proceso creativo y en el objeto artístico mismo.

Así, para la generación de conocimiento en las artes será imprescindible que se documente con variados formatos el proceso de asimilación no convencional de los visitantes. Para esto, debe haber una comunicación clara entre el personal que facilite la práctica, y los visitantes, así como el personal institucional (en el caso de que el personal que facilita sea externo al museo). Dentro de los registros, el registro verbal de la narrativa del visitante es muy valioso para una evaluación durante y a posteriori. Además, la conformación de un archivo como registro investigativo que valide el proceso es uno de los fines de esta propuesta metodológica.

\section{Mutabilidad y adaptabilidad:}

La experimentación se caracteriza precisamente por la imposibilidad de establecer un método estricto no modificable. Esta propuesta, si bien presenta ejemplos internacionales, deberá ser posible de adecuar a cualquier contexto, especialmente el latinoamericano. Es necesario reconocer la multiplicidad de contextos y poner énfasis en la localidad, singularidad y conocimientos autóctonos. Así, una práctica museológica experimental ideal tomaría en cuenta el acceso a tecnologías, el contexto diario del grupo de visitantes y, en cierta medida, el pasado histórico del museo.

\section{Agencia en la Museología Experimental}

La segunda relación importante para la museología experimental tiene que ver con la agencia del dispositivo; el museo como un dispositivo social con agencia (Brulon Soares, 2017). Como bien lo explica Brulon Soares, corresponde con el tercer principio de incertidumbre de Bruno Latour (2005, p.64): los objetos también tienen agencia.

Si las inequidades deben ser generadas, esto es prueba de que otros tipos de actores aparte de los sociales entran en juego. ${ }^{.}$

Además, establece que el museo tiene una agencia sobre las experiencias humanas. Se pone énfasis en lo social del museo como una fuerza especifica. Latour (2005, p.66) explica como las interacciones temporales, inmediatas o

\footnotetext{
I. "if inequalities have to be generated, this is proof that other types of actors than the social ones are coming into play."
} 
superficiales, se pueden convertir en interacciones de largo alcance, profundas, durables y significativas.

El potencial, tanto de la sociabilidad como de la agencia, residirá en la capacidad de crear experiencias significativas con fuerza social que nutran las realidades de los visitantes. Esta propuesta, busca que la agencia no sea solo implementada por el museo, sino que los visitantes comprendan que también tienen capacidad de agencia, justamente a través de la creación de dispositivos en actividades guiadas.

Una museología experimental animaría a los visitantes, a pensar en su posición personal respecto al tema expositivo. Por eso el interés de que fabriquen un objeto que propicie agencia, permitiéndoles deconstruir la exposición, crear y compartir su versión de realidad personal en paralelo a la narrativa que propone el museo.

Dicho objeto vendría a ser una especie de Artefacto cognitivo inteligente (Varela, Thompson y Rosch, I992, p. 24I). Sería ideal disolver las barreras entre la institución, los visitantes y los objetos, justamente a través del pensamiento crítico y la acción creativa, buscando alcanzar una horizontalidad. Antes de adentrarnos en el tema de fabricación de dispositivos es valioso analizar brevemente un par de ejemplos de tecnologías interactivas recientes.

Un ejemplo - quizás tecno utópico y alejado de la realidad Latinoamericana que podría acercarse a las propuestas anteriormente descritas se encuentra en el área de Koto-ku, en Tokio, Japón. Mori Building Digital Art Museum (Museo de Arte Digital Edificio Mori), TeamLab Borderless, presenta una experiencia inmersiva con diferentes aplicaciones de realidad aumentada, ${ }^{\mathrm{I}}$ en distintas líneas narrativas, donde las obras interactivas digitales se interconectan e influencian entre sí, sincronizadas por medio de algoritmos generativos y reaccionando según la ubicación y número de visitantes. Cada persona que visite el museo, tendrá una experiencia distinta, única e irrepetible.

Esta es principalmente, una experiencia estética enfocada en el entretenimiento. Es decir, las temáticas buscan principalmente el goce estético, y el aprendizaje lúdico y participativo. Algunas de las salas ofrecen actividades educativas e interactivas. Nos enfocaremos en una de estas actividades.

\footnotetext{
I. Realidad aumentada y realidad virtual: El término realidad virtual, fue acunado oficialmente en el año 1987 por Jaron Lanier, fundador del Visual Programming Lab, dándole nombre por fin al área de investigación que estudia la realidad virtual definida como la creación de un medio artificial donde ilusoriamente nos sentimos presentes. (Virtual Reality Society, 20I6, vrs.org.uk). Más ampliamente, Virtual Reality Society, estipula: "La definición de realidad virtual, proviene, naturalmente, de las definiciones combinadas entre los vocablos "virtual" y "real". "Virtual" significa "cercano" y "realidad" es lo que experimentamos como seres humanos. Así, el término realidad virtual, básicamente significa "cercano a la realidad". Esto podría, por supuesto, significar cualquier cosa, pero usualmente se refiere a un tipo específico de emulación de realidad." (Virtual Reality Society, 2or6, vrs.org.uk). (Trad de M. Aguilar 2018)
} 
En el área del Bosque Atlético (Athletic Forest), una de las salas adyacentes a la sala principal, ofrece un espacio con mesas y materiales de dibujo donde los visitantes pueden elegir una silueta que representa una criatura marina (varios tipos de peces, calamar, pulpo, tortuga, caballito de mar) para colorearla, escanearla y agregarla a la instalación digital interactiva (mural animado) proyectado en las paredes de la sala; una escena subacuática que se crea a partir de las colaboraciones de los visitantes y donde los dibujos se mueven según una secuencia pre programada.

La actividad promueve la participación colectiva. Según los diseñadores de la experiencia, se nutren habilidades de creatividad, poder de expresión, respeto a la diversidad, interés en la tecnología y consciencia espacial. Los visitantes tienen capacidad de decisión sobre el diseño de su ilustración. Sin embargo, no deciden sobre la forma o la secuencia de movimiento de la misma. No hay agencia, en ese sentido y ello se ve limitado por la tecnología utilizada. Es decir, el poder de decisión de los visitantes termina cuando entra en juego el dispositivo computacional, y más especificamente, cuando la imagen física es digitalizada por el escaner.

Otra de las desventajas de esta aplicación es que no hay un mecanismo de evaluación in situ que archive la experiencia. Borderless tampoco ofrece un registro para los visitantes sobre la animación. Una vez depositada en el mar digital, no hay posibilidad de continuación de la experiencia en otra plataforma y teamLab Borderless se apropia con los derechos de autoría de las imágenes. Los visitantes dependen de sus registros personales con sus dispositivos móviles y únicamente se llevan sus dibujos a casa. ¿Puede esta ser catalogada como una experiencia educativa significativa si no hay más seguimiento de parte del personal que el escaneo de los dibujos y los pocos datos proporcionados en la hoja para el dibujo? Hay una clara diferencia entre participación y agencia. Este ejemplo es muestra de ello.

Un segundo ejemplo, esta vez aplicado en contexto Centroamericano, es el proyecto Uramado: Tanukis Awake, por la artista multimedia Julie S. Chheng. Fue presentado como parte de las actividades de la Noche en Blanco (Nuit Blanche, Art City Tour) en noviembre del 2019 a cargo de la Alianza Francesa en Costa Rica. Fue la primera vez que el proyecto se presentó en latinoamérica y es de las primeras propuestas de realidad aumentada en espacios públicos que forma parte de una actividad cultural en el país.

Uramado: Tanukis Awake es una ruta de búsqueda del tesoro en realidad aumentada que cuenta la historia de los Tanukis (espíritus guardianes del bosque que se despiertan en la ciudad). Los Tanukis se ubican en diferentes lugares de un área delimitada, un tanto ocultos y son de distintos tamaños (Chheng, 2019). Los participantes deben ir encontrándolos con sus dispositivos móviles en una aplicación de descarga gratuita y respondiendo a las formuladas por los personajes animados. Ello revela rasgos de la personalidad del Tanuki y contribuye a asignar un Tanuki al participante como recompensa al completar la búsqueda. 
La ruta ha sido activada en diferentes ubicaciones geográficas como Tokyo, Kyoto, Nueva York, Francia y más recientemente en Costa Rica.

El público fue invitado vía redes sociales y prensa a estar atentos a los animales escondidos en distintos rincones del Área Metropolitana costarricense y descargar la aplicación gratuita en sus dispositivos móviles.

El 2r de Noviembre del 2019, día de la celebración de la Noche en Blanco, Llegaron alrededor de setecientas personas a la sede de la Alianza Francesa. Recibieron la inducción sobre el uso de la aplicación móvil y las reglas del juego. $\mathrm{Al}$ completar la búsqueda, los participantes regresaban a la Alianza Francesa a recolectar la máscara correspondiente al Tanuki asignado. Los participantes del Art City Tour, también podían conocer sobre el juego en dos de los puntos de mayor afluencia (entradas principales del Museo de Arte y Diseño Contemporáneo y de Museos del Banco Central), por medio de afiches informativos y de los guías del Art City Tour.

La dinámica, además de tener una formulación lúdica y colaborativa, permitía una apropiación del espacio público citadino y la generación de nuevas relaciones con el entorno. Simbólicamente, la máscara Tanuki personalizada asignada como recompensa al completar el juego funciona como un objeto que determina a su portador como guardián de la naturaleza; constituyéndose esta en una característica significativa y de gran importancia para todo el desarrollo del juego.

En el caso de Uramado: Tanukis Awake, los participantes de la experiencia no tienen una agencia sobre la tecnología; sin embargo, funciona como una opción para recorrer espacios expositivos experimentalmente e indirectamente influenciar en la percepción de las exposiciones del Art City Tour. Es decir, Uramado funciona como estrategia transmedia para vincular narrativas en diferentes espacios y es ejemplo de la cognición como acción corporizada.

\section{Propuesta metodológica}

En el inicio del texto propusimos una asimilación no convencional como herramienta de la museología experimental. El circuito de asimilación normal de los visitantes podría tornarse en una asimilación no convencional de la siguiente manera:

Una chispa de curiosidad atrapa la atención del sujeto; el sujeto encuentra una parte del objeto de atención; el sujeto se enlaza en una interacción con el objeto que llama su atención y lo revela por completo. Durante este proceso inicia una relación con el universo de precognición del sujeto.

O bien:

El sujeto colabora con otros como colectivo, es posible construir, recibir o conocer el objeto completo sólo a través de esta acción conjunta. Los universos de precognición y los bagajes de cada individuo contribuyen a la nueva 
significación o valoración del objeto. Esta nueva información abre un abanico de posibilidades o ideas capaces de transformar las historias en la vida del sujeto, su percepción de realidad y, por tratarse de un grupo de personas, se conformará una comunidad temporal no condicionada. (Groys, 20I4, p. 6o-6I).

Con esta idea general toda actividad propuesta sería inmersiva, multisensorial (involucrando al menos dos sentidos), lúdica y con dinámicas activas. Además, tendría que ser documentada y compartida en alguna forma digital como archivo e idealmente presentarse como una extensión de la narrativa generada por el museo como transmedia.

Actualmente se está organizando un piloto que cumpla estos requerimientos. La propuesta está vinculada a la obra denominada Khipu, de la artista chilena Constanza Piña Pardo (obra presentada en la más reciente edición del festival de arte y tecnología Ars Electrónica, con sede en Linz, Austria).

Khipu, es una instalación interactiva que recrea visual y tecnicamente los objetos del mismo nombre que los antiguos Incas utilizaban para almacenar información. Estos dispositivos consistían en cuerdas con nudos de distintos tipos y colores para almacenar datos codificados. Son considerados instrumentos mnemotécnicos y computadores ancestrales. La obra de Pardo integra la tradición ancestral con nuevas tecnologías, mediante la sonificación del Khipu usando un circuito análogo.

La intención es generar una actividad con un grupo cautivo de visitantes idealmente durante uno o dos días completos. Primero habría una visita guiada a la exposición, la guía pondrá énfasis en aspectos clave de Khipu para ser analizados posteriormente.

Seguidamente habría un taller de creación donde los visitantes fabrican su propio dispositivo. Este dispositivo será algún objeto que permita ampliar la percepción de la instalación con otros sentidos. Los objetos fabricados podrían ser antenas, detectores de campo electromagnético (ampliación o variación por medio del sonido), objetos análogos que modifiquen la percepción visual de la instalación (lentes especiales, realidad aumentada), etc. Su fabricación deberá fomentar en los visitantes - ahora participantes - su capacidad de crear tecnologías que apoyen alguna interacción humano-objeto (en este caso el objeto de arte). Los objetos serán de bajo costo, accesibles y de código abierto. En el caso de herramientas digitales, se intentará en lo posible usar software libre.

Tras el taller se haría una segunda visita a la exposición para que los visitantes prueben sus objetos. Habría una evaluación de su experiencia, de cómo cambió antes y después de conocer la obra con los dispositivos. Se harían registros de estas narraciones tanto para ser evaluadas por facilitadores y como contenido para el archivo del proyecto.

A partir de la finalización del taller, se deja abierta la posibilidad de vinculación de visitantes con la experiencia por medio de la plataforma digital que las archiva. Esta plataforma sería alojada en un servidor en un router indepen- 
dientes. El servidor y el router garantizarían acceso a los visitantes y el hecho no pertenecer a dominios privativos virtuales constituye una afirmación de la agencia tanto del museo como del proyecto en sí mismo. La extensión del proyecto en el mundo virtual es también correspondiente con la práctica museológica experimental.

\section{Conclusiones}

Este modo horizontal de trabajar demanda que artistas y críticos conozcan no sólo la estructura de cada cultura lo bastante bien como para mapearla, sino también su historia como para narrarla. (..) Coordinar ambos ejes de varios de tales discursos supone una carga enorme. Y aquí debe considerarse, aunque no sea más que para contrarrestarla, la cautela tradicionalista respecto al modo horizontal de trabajar: que las nuevas conexiones discursivas puedan difuminar los viejos recuerdos disciplinarios. (Foster, 1996, p. 270)

\section{9}

En este caso a la lista incluiría también a los museólogos en un afán por trabajar horizontalmente con los visitantes del museo. Se reitera la importancia del museo como institución no solo para el entretenimiento, sino como medio para la concientización de realidades político-sociales y como potencial contenedor para despertar a la acción desde la creatividad. La museología experimental, buscaría contribuir de manera real y práctica con la sociedad y esa horizontalidad implica la apertura transdisciplinar, la disolución de jerarquías institución-artista-visitante; es decir, la deconstrucción del aparato museal tradicional.

La búsqueda de nuevas conexiones o prácticas museológicas experimentales implica en el contexto Latinoamericano una imperante necesidad de generar conocimientos, como lo expresa Galindo Monteagudo (2019, pp. 56):

Como los libros que nos llegan en su mayoría extranjeros, esto nos hacen conocer mucho más el desarrollo de esta ciencia en Europa y Estados Unidos e incluso en Oceanía, ya que la producción en lengua inglesa es abundante, también nos llega mucha producción española, pero es poco lo que se de la producción realizada en este país y las perspectivas investigadas en América Latina, Asia y África.

Esa deficiencia en el conocimiento a nivel regional, se debe ver como una oportunidad para la proposición y experimentación. Este proyecto en curso intenta motivar a la comunidad de museólogas y museólogos latinoamericanos 
a contribuir en nuestro campo de conocimiento con formas activas, propositivas y nuevas.

\section{Referencias}

Borgdorff, H. (20I0). El debate sobre la investigación en las artes. CAIRON. Revista de estudios de danza / Journal of Dance Studies. Universidad de Alcalá, I3, 25-46. Recuperado el I7 marzo 2020 de: http://archivoartea. uclm.es/wp-content/uploads/2018/ı2/cairon-I3.pdf

Brulon Soares, B. (2017). Le musée experimental: definir le mouvement, apprehender le passage. En Francois Mairesse (Ed.) Definir le musee du XXIe siecle. Materiaux pour une discussion. Paris: ICOFOM.

Chheng, J. Uramado AR. (2019). Recuperado el 3 marzo 2020 de: https://juliestephenchheng.com/portfolio/kayak-ar/

Di Paolo, E. A. (20r3). El enactivismo y la naturalización de la mente. En D. P. Chico and M. G. Bedia (Ed.), Nueva ciencia cognitiva: Hacia una teoría integral de la mente. Madrid, España: Plaza y Valdes.

Documenta gGmbH. (2020). About. Documenta gGmbH. Recuperado el 8 de marzo 2020 de: https://www.documenta.de/en/about\#I6_documenta_ ggmbh

Foster, H. (1996). The Return of the Real: The Avant-Garde at the End of the Century, Massachusetts Estados Unidos: MIT Press.

Galindo Monteagudo, S.R. (2019). El futuro de la tradición Museológica. En K. Smeds (Ed.), The Future of Tradition in Museology. Materials for a discussion. Papers from the ICOFOM 42nd symposium held in Kyoto (Japan), 1-7 September 2019 (pp. 55-59). Paris: ICOFOM.

Groys, B. (2014). Volverse Público. Las Transformaciones del Arte en el Ágora Contemporánea. Buenos Aires Argentina: Caja Negra.

Latour, B. (2005). Reassembling the Social. Introduction to Actor Network Theory. Oxford, Inglaterra: Oxford University Press.

Piaget, J. (1952). The Origins of Intelligence in Children. New York: International Universities Press, Inc.

Schmitt, D. (2016). Pour une approche énactive de la museologie. ICOFOM Study Series, 44, I07-II4.

Schmitt, D., \& Aubert, O. (20I6) REMIND : une méthode pour comprendre la micro-dynamique de l'expérience des visiteurs de musées. Revue des Interactions Humaines Médiatisées, Europia, I7 (2), 43-70. 
Varela, F., Thompson, E., \& Rosch, E. (I992). De cuerpo presente. Barcelona, España: Gedisa.

Varela, F., \& Maturana, H. (1994). El árbol del conocimiento. Raíces biológicas de la comprensión humana. Paris, Francia: Addison-Wesley.

Virtual Reality Society. History of Virtual Reality. (2016) Virtual Reality Society recuperado el 5 febrero 2017 de: http://www.vrs.org.uk/virtual-reality/ history.html 
Papers - Museología Experimental. Hacia un método práctico 